\title{
CHARACTERIZATION OF NASH EQUILIBRIUM STRATEGY FOR HEPTAGONAL FUZZY GAMES
}

\author{
F. MADANDAR ${ }^{1}$, S. HAGHAYEGHI ${ }^{1}$ AND S. M. VAEZPOUR ${ }^{2, *}$ \\ ${ }^{1}$ Department of Mathematics, Karaj Branch, Islamic Azad University, Karaj, Iran \\ ${ }^{2}$ Department of Mathematics and Computer Science, Amirkabir University of Technology, Tehran, Iran \\ *Corresponding author: vaez@aut.ac.ir

\begin{abstract}
In this paper, the Nash equilibrium strategy of two-person zero-sum games with heptagonal fuzzy payoffs is considered and the existence of Nash equilibrium strategy is studied. Also, based on the fuzzy max order several models in heptagonal fuzzy environment is constructed and the existence of their equilibrium strategies is proposed. In the sequel, the existence of Pareto Nash equilibrium strategies and weak Pareto Nash equilibrium strategies is investigated for fuzzy matrix games. Finally, the relation between Pareto Nash equilibrium strategy and parametric bi-matrix games is established.
\end{abstract}

\section{INTRODUCTION}

Modern game theory was developed by the mathematician John Von Neumann in the Mid-1940's and in 1944, he published the book of "Theory of games and economic behavior" joint with Morgenstern [9]. The most important categories are as cooperative and non-cooperative games. In 1951, non-cooperative games was presented by John Nash [8]. In this article we focus on a class of non-cooperative games namely two-person zero-sum matrix games. Moreover, one of the most important concepts in game theory is the Nash equilibrium. Nash proves that if we approve mixed strategies, then every game with a finite number of players has at least one Nash equilibrium.

Received 2017-12-13; accepted 2018-02-05; published 2018-05-02.

2010 Mathematics Subject Classification. 91A05.

Key words and phrases. zero-sum matrix game; fuzzy payoffs; Nash equilibrium; heptagonal fuzzy number.

(c)2018 Authors retain the copyrights of their papers, and all open access articles are distributed under the terms of the Creative Commons Attribution License. 
In 1978, the idea of fuzziness was exhibited by Zadeh [15], that is a type of subjective uncertainty. After then fuzzy numbers found many applications in various fields with incomplete information such as engineering, social and economics. In many science such as economics, business competition, auction and etc., the payoff for games is not realistic indeed the payoffs are fuzzy.

Many of mathematicians and reseachers study the fuzziness. Zimmermann [16] in 1985, Yazenin [13] in 1987 and Sakawa [12] in 1993 applied the fuzzy theory to optimization problems. In 1989, Campos [2] transformed the fuzzy games into fuzzy optimization problems. In 1999, Liu [5] founded theory in the uncertain environments. In 2000, Meada [7] constructed kind of concepts of minimax equilibrium strategies. In 2005, C. R. Bector and S. Chandra [1] provided fuzzy mathematical programming and fuzzy matrix games. In 2011, Cunlin and Zhang Qiang [4] investigated two-person zero-sum games in the symmetric triangular fuzzy environment. They obtained Nash equilibrium of two-person zero-sum games with fuzzy payoff. They also obtained Pareto Nash equilibrium strategy for fuzzy matrix game. In 2014, Bapi Dutta [3] extended their work in trapezoidal fuzzy environment and he introduced two special types of fuzzy games: constant and proportional fuzzy game. In $[3,4,7]$ the uncertainty and imprecision in payoffs have been represented by either triangular or trapezoidal fuzzy numbers.

The most frequently used fuzzy numbers in the different problems are triangular or trapezoidal fuzzy numbers. But, it is not possible to restrict the membership functions to take either triangular or trapezoidal form. Therefore this paper focus on fuzzy payoffs of decision makers by heptagonal fuzzy numbers. In 2014, the arithmetic operations of heptagonal fuzzy numbers are defined by K. Rathi and S. Balamohan [10]. The heptagonal fuzzy number gives additional possibility to represent imperfect knowledge what leads to model many problems. Heptagonal fuzzy numbers have different applications in optimization problems and decision making problems which need seven parameters.

In this paper we define the $k$-heptagonal fuzzy numbers and generalize Cunlin and Qiang [4] and Bapi Dutta [3] Nash equilibrium solution concepts. The paper is organized as follows: In section 2, the basic definitions and notations of fuzzy numbers are given. In section 3, we introduce the notation of two-person zero-sum matrix games with heptagonal fuzzy payoffs, the different types of equilibrium strategies and investigate their existence conditions for the fuzzy games. In section 4, parametric bi-matrix games are introduced and then the relation between parametric bi-matrix games and Nash equilibrium strategies is studied. In section 5 , we present some illustrative exampes.

\section{Preliminaries}

In this section, we suggest some basic definitions and concepts of fuzzy numbers, which were proposed by Zadeh [14] in 1965. Also, we introduce some notations of fuzzy sets, such as $\alpha$-cut for heptagonal fuzzy numbers. 
Definition 2.1. [4] A fuzzy number $\tilde{a}$ is a fuzzy set on the real line $\mathbb{R}$ if its membership function $\mu_{\tilde{a}}(x)$ satisfies the following conditions.

(i) $\mu_{\tilde{a}}(x)$ is a mapping from $\mathbb{R}$ to the closed interval [0, 1];

(ii) There exist a unique real number $c$, called the center of $\tilde{a}$, such that ;

(a) $\mu_{\tilde{a}}(c)=1$;

(b) $\mu_{\tilde{a}}(x)$ nondecreasing on $(-\infty, c]$;

(c) $\mu_{\tilde{a}}(x)$ nonincreasing on $[c,+\infty)$.

The $\alpha$-cut or $\alpha$-level of fuzzy number have an important role in parametric ordering of fuzzy numbers. The $\alpha$-cut set of a fuzzy number $\tilde{a}$, denoted by $[\tilde{a}]_{\alpha}$. Every $\alpha$-cut is a closed interval $[\tilde{a}]_{\alpha}=\left[a_{\alpha}^{L}, a_{\alpha}^{U}\right] \subset \mathbb{R}$, where $a_{\alpha}^{L}=\inf \left\{x \in \mathbb{R} \mid \mu_{\tilde{a}}(x) \geq \alpha\right\}$ and $a_{\alpha}^{U}=\sup \left\{x \in \mathbb{R} \mid \mu_{\tilde{a}}(x) \geq \alpha\right\}$ for any $\alpha \in[0,1]$. For more details see $[4,6]$.

Definition 2.2. A fuzzy number $\tilde{a}=(a, c, b, h, l, r, m)$ is called a $k$-heptagonal if its membership function is defined as

$$
\mu_{\tilde{a}}(x)= \begin{cases}k\left(\frac{x-a+h}{h}\right) & , a-h \leq x \leq a, \\ k & , a \leq x \leq c-l, \\ k+\left(\frac{x-c+l}{l}\right) & , c-l \leq x \leq c, \\ k+\left(\frac{c+r-x}{r}\right) & , c \leq x \leq c+r \\ k & , c+r<x<b+m, \\ 0 & , \text { otherwise. }\end{cases}
$$

where $c$ is the center of $\tilde{a}$ and $h, l, r, m$ are non-negative.

In the rest of the paper, for simplicity, the $k$-heptagonal fuzzy number is denoted by $k$-HFN.

Let $\tilde{a}=\left(a_{1}, c_{1}, b_{1}, h_{1}, l_{1}, r_{1}, m_{1}\right)$ and $\tilde{b}=\left(a_{2}, c_{2}, b_{2}, h_{2}, l_{2}, r_{2}, m_{2}\right)$ are two $k$-HFN then

- Addition:

$$
\tilde{a}+\tilde{b}=\left(a_{1}+a_{2}, c_{1}+c_{2}, b_{1}+b_{2}, h_{1}+h_{2}, l_{1}+l_{2}, r_{1}+r_{2}, m_{1}+m_{2}\right)
$$

- Subtraction:

$$
\tilde{a}-\tilde{b}=\left(a_{1}-m_{2}, c_{1}-r_{2}, b_{1}-l_{2}, h_{1}-h_{2}, l_{1}-b_{2}, r_{1}-c_{2}, m_{1}-a_{2}\right)
$$

- Scalar Multiplication :

$$
\begin{gathered}
\lambda>0, \lambda \in \mathbb{R} ; \lambda \tilde{a}=\left(\lambda a_{1}, \lambda c_{1}, \lambda b_{1}, \lambda h_{1}, \lambda l_{1}, \lambda r_{1}, \lambda m_{1}\right) \\
\lambda<0, \lambda \in \mathbb{R} ; \lambda \tilde{a}=\left(\lambda m_{1}, \lambda r_{1}, \lambda l_{1}, \lambda h_{1}, \lambda b_{1}, \lambda c_{1}, \lambda a_{1}\right) .
\end{gathered}
$$


By definition of $\alpha$-cut we have the following lemma.

Lemma 2.1. Let $\tilde{a}=\left(a_{1}, c_{1}, b_{1}, h_{1}, l_{1}, r_{1}, m_{1}\right)$ be a $k$-HFN. Then for $\alpha \in(0,1]$, the $\alpha$-cut of $\tilde{a}$ is,

$$
[\tilde{a}]_{\alpha}=\left\{\begin{array}{l}
{\left[a-\left(1-\frac{\alpha}{k}\right) h, b+\left(1-\frac{\alpha}{k}\right) m\right] ; \alpha \in(0, k]} \\
{\left[c-\left(\frac{1-\alpha}{1-k}\right) l, c+\left(\frac{1-\alpha}{1-k}\right) r\right] ; \alpha \in[k, 1] .}
\end{array}\right.
$$

Definition 2.3. [4] Let $x=\left(\xi_{1}, \xi_{2}, \ldots, \xi_{n}\right)$ and $y=\left(\eta_{1}, \eta_{2}, \ldots, \eta_{n}\right)$ be vectors in $\mathbb{R}^{n}$. Then

(i) $x \geqq y$ if and only if $\xi_{i} \geq \eta_{i}$ for all $i=1,2, \ldots, n$,

(ii) $x \geq y$ if and only if $x \geqq y$ and $x \neq y$,

(iii) $x>y$ if and only if $\xi_{i}>\eta_{i}$ for all $i=1,2, \ldots, n$.

Definition 2.4. [4] Let $\tilde{a}$ and $\tilde{b}$ be two fuzzy numbers. Then,

(i) $\tilde{a} \succsim \tilde{b}$ if and only if $\left(a_{\alpha}^{L}, a_{\alpha}^{U}\right) \geqq\left(b_{\alpha}^{L}, b_{\alpha}^{U}\right)$, for all $\alpha \in[0,1]$,

(ii) $\tilde{a} \succsim \tilde{b}$ if and only if $\left(a_{\alpha}^{L}, a_{\alpha}^{U}\right) \geq\left(b_{\alpha}^{L}, b_{\alpha}^{U}\right)$, for all $\alpha \in[0,1]$,

(iii) $\tilde{a} \succ \tilde{b}$ if and only if $\left(a_{\alpha}^{L}, a_{\alpha}^{U}\right)>\left(b_{\alpha}^{L}, b_{\alpha}^{U}\right)$, for all $\alpha \in[0,1]$.

The following theorem characterize the orders for heptagonal fuzzy numbers.

Theorem 2.1. Let $\tilde{a}=\left(a_{1}, c_{1}, b_{1}, h_{1}, l_{1}, r_{1}, m_{1}\right), \tilde{b}=\left(a_{2}, c_{2}, b_{2}, h_{2}, l_{2}, r_{2}, m_{2}\right)$ be two $k$-HFN. Then

(i) $\tilde{a} \precsim \tilde{b}$ if and only if

$$
\begin{aligned}
& \max \left\{h_{2}-h_{1}, 0\right\} \leq a_{2}-a_{1}, \max \left\{m_{1}-m_{2}, 0\right\} \leq b_{2}-b_{1}, \\
& \max \left\{l_{2}-l_{1}, 0\right\} \leq c_{2}-c_{1} \text { and } \max \left\{r_{1}-r_{2}, 0\right\} \leq c_{2}-c_{1},
\end{aligned}
$$

(ii) $\tilde{a} \prec \tilde{b}$ if and only if

$$
\begin{aligned}
& \max \left\{h_{2}-h_{1}, 0\right\}<a_{2}-a_{1}, \max \left\{m_{1}-m_{2}, 0\right\}<b_{2}-b_{1}, \\
& \max \left\{l_{2}-l_{1}, 0\right\}<c_{2}-c_{1} \text { and } \max \left\{r_{1}-r_{2}, 0\right\}<c_{2}-c_{1} .
\end{aligned}
$$

Proof. By using Definition (2.4) $\tilde{a} \precsim \tilde{b}$ if and only if for all $\alpha \in[0,1]$, $\left(a_{\alpha}^{L}, a_{\alpha}^{U}\right) \leqq\left(b_{\alpha}^{L}, b_{\alpha}^{U}\right)$ or equivalently $a_{\alpha}^{L} \leqslant b_{\alpha}^{L} \quad$ and $\quad a_{\alpha}^{U} \leqslant b_{\alpha}^{U}$. But by Lemma $(2.1) a_{\alpha}^{L} \leqslant b_{\alpha}^{L}$ if and only if $a_{1}-\left(1-\frac{\alpha}{k}\right) h_{1} \leqslant a_{2}-\left(1-\frac{\alpha}{k}\right) h_{2}$ for all $\alpha \in[0, k]$ and $c_{1}-\left(\frac{1-\alpha}{1-k}\right) l_{1} \leqslant c_{2}-\left(\frac{1-\alpha}{1-k}\right) l_{2}$ for all $\alpha \in[k, 1]$, which are equivalent to

$$
\left(1-\frac{\alpha}{k}\right)\left(h_{2}-h_{1}\right) \leqslant a_{2}-a_{1} \text { for all } \alpha \in[0, k]
$$

and

$$
\left(\frac{1-\alpha}{1-k}\right)\left(l_{2}-l_{1}\right) \leqslant c_{2}-c_{1} \text { for all } \alpha \in[k, 1] .
$$

which are equivalent to $\max \left\{h_{2}-h_{1}, 0\right\} \leq a_{2}-a_{1}$ and $\max \left\{m_{1}-m_{2}, 0\right\} \leq b_{2}-b_{1}$. Similarly, by using Lemma(2.1) it can be conclude $a_{\alpha}^{U} \leqslant b_{\alpha}^{U}$ if and only if $\max \left\{l_{2}-l_{1}, 0\right\} \leq c_{2}-c_{1}$ and $\max \left\{r_{1}-r_{2}, 0\right\} \leq c_{2}-c_{1}$ and the proof of part $(i)$ is complete. Part $(i i)$ can be proved, similarly. 


\section{Two-Person Zero-sum Matrix FuzZy Games}

In this section, we shall consider two-person zero-sum games with fuzzy payoffs. Let $P=\{1,2, \ldots, p\}$ and $Q=\{1,2, \ldots, q\}$ be the sets of pure strategies of player $I$ and player $I I$, respectively. Let $A=\left(a_{i j}\right)_{p \times q}$ be the payoff matrix whose entries $a_{i j}$ denote the payoff that player $I$ gains and player $I I$ loses. In the zero-sum games $-a_{i j}$ is the amount paid by player $I$ to player $I I$ i.e. the gain of one player is the loss of other player. The mixed strategies of players $I$ and player $I I$ are probability distributions on the set of pure strategies, represented by

$$
\begin{aligned}
& X=\left\{\left(\xi_{1}, \xi_{2}, \ldots, \xi_{p}\right) \in \mathbb{R}^{p} \mid \xi_{i} \geq 0, i=1,2, \ldots, p, \sum_{i=1}^{p} \xi_{i}=1\right\} \\
& Y=\left\{\left(\eta_{1}, \eta_{2}, \ldots, \eta_{q}\right) \in \mathbb{R}^{q} \mid \eta_{j} \geq 0, i=1,2, \ldots, q, \sum_{j=1}^{q} \eta_{j}=1\right\} .
\end{aligned}
$$

respectively.

In this section, the payoffs of the pair $(x, y) \in X \times Y$ are modeled by $k$-heptagonal fuzzy number $\tilde{a}=$ $(a, c, b, h, l, r, m)$. Let player $I$ choose a mixed strategy $x \in X$ and player $I I$ choose mixed strategy $y \in Y$. The $k$-heptagonal fuzzy number $\tilde{a}_{i j}=\left(a_{i j}, c_{i j}, b_{i j}, h_{i j}, l_{i j}, r_{i j}, m_{i j}\right)$ indicates the payoffs that player $I$ receives and player $I I$ loses, the fuzzy payoff matrix of the game is given by

$$
\tilde{A}=\left(\begin{array}{ccc}
\tilde{a}_{11} & \cdots & \tilde{a}_{1 q} \\
\vdots & \ddots & \vdots \\
\tilde{a}_{p 1} & \cdots & \tilde{a}_{p q}
\end{array}\right) .
$$

The fuzzy two-person zero-sum games is denoted by $\tilde{\Gamma} \equiv(X, Y, \tilde{A})$. The fuzzy payoffs of the players $I$ and II are

$$
\begin{aligned}
x^{T} \tilde{A} y & =\sum_{i=1}^{p} \sum_{j=1}^{q} \xi_{i} \tilde{a}_{i j} \eta_{j} \\
& =\left(x^{T} A y, x^{T} C y, x^{T} B y, x^{T} H y, x^{T} L y, x^{T} R y, x^{T} M y\right),
\end{aligned}
$$

which is a $k$-heptagonal fuzzy number, for more details see [11]. In the rest of this paper, we set $\tilde{A}=$ $\left(\tilde{a}_{i j}\right), A=\left(a_{i j}\right), C=\left(c_{i j}\right), B=\left(b_{i j}\right), H=\left(h_{i j}\right)$,

$R=\left(r_{i j}\right), M=\left(m_{i j}\right)$, where $\tilde{A}, A, B, H, L, R$ and $M$ are $p \times q$ matrix. Also $\tilde{A}$ is a fuzzy $k$-heptagonal payoff matrix.

Definition 3.1. [3] A pair $\left(x^{*}, y^{*}\right) \in X \times Y$ is called a Nash equilibrium strategy for a game $\tilde{\Gamma}$ if

(i) $x^{T} \tilde{A} y^{*} \precsim x^{* T} \tilde{A} y^{*}, \forall x \in X$,

(ii) $x^{* T} \tilde{A} y^{*} \precsim x^{* T} \tilde{A} y, \forall y \in Y$. 
Theorem 3.1. Let $\tilde{\Gamma}=(X, Y, \tilde{A})$ be a two-person zero-sum game with fuzzy payoffs, the pair $\left(x^{*}, y^{*}\right)$ is the expected Nash Equilibrium strategy of $\tilde{\Gamma}$ if and only if :

(i) $x^{T} A y^{*} \leqslant x^{* T} A y^{*} \leqslant x^{* T} A y$,

(ii) $x^{T} B y^{*} \leqslant x^{* T} B y^{*} \leqslant x^{* T} B y$,

(iii) $x^{T} C y^{*} \leqslant x^{* T} C y^{*} \leqslant x^{* T} C y$,

(iv) $x^{T}(A-H) y^{*} \leqslant x^{* T}(A-H) y^{*} \leqslant x^{* T}(A-H) y$,

(v) $x^{T}(B-L) y^{*} \leqslant x^{* T}(B-L) y^{*} \leqslant x^{* T}(B-L) y$,

(vi) $x^{T}(B+R) y^{*} \leqslant x^{* T}(B+R) y^{*} \leqslant x^{* T}(B+R) y$,

(vii) $x^{T}(C+M) y^{*} \leqslant x^{* T}(C+M) y^{*} \leqslant x^{* T}(C+M) y$.

Proof. Let $\tilde{\Gamma}$ be a two-person zero-sum game with the fuzzy $k$-heptagonsl payoff matrix $\tilde{A}=(A, C, B, H, L, R, M)$. Let $\left(x^{*}, y^{*}\right) \in X \times Y$ be the Nash equilibrium strategy of the game $\tilde{\Gamma}$. Therefore by Definition (3.1) we have

$$
x^{* T} \tilde{A} y^{*} \precsim x^{* T} \tilde{A} y, \forall y \in Y .
$$

Since

$$
x^{T} \tilde{A} y^{*}=\left(x^{T} A y^{*}, x^{T} C y^{*}, x^{T} B y^{*}, x^{T} H y^{*}, x^{T} L y^{*}, x^{T} R y^{*}, x^{T} M y^{*}\right),
$$

and

$$
x^{* T} A y^{*}=\left(x^{* T} A y^{*}, x^{* T} C y^{*}, x^{* T} B y^{*}, x^{* T} H y^{*}, x^{* T} L y^{*}, x^{* T} R y^{*}, x^{* T} M y^{*}\right),
$$

So, by Theorem (2.1), $x^{T} \tilde{A} y^{*} \precsim x^{* T} \tilde{A} y^{*}$ if and only if

$$
\begin{array}{r}
\max \left\{x^{* T} H y^{*}-x^{T} H y^{*}, 0\right\} \leqslant x^{* T} A y^{*}-x^{T} A y^{*}, \\
\max \left\{x^{T} M y^{*}-x^{* T} M y^{*}, 0\right\} \leqslant x^{* T} B y^{*}-x^{T} B y^{*}, \\
\max \left\{x^{* T} L y^{*}-x^{T} L y^{*}, 0\right\} \leqslant x^{* T} C y^{*}-x^{T} C y^{*}, \\
\max \left\{x^{T} R y^{*}-x^{* T} R y^{*}, 0\right\} \leqslant x^{* T} C y^{*}-x^{T} C y^{*} .
\end{array}
$$

Consequently $x^{T} \tilde{A} y^{*} \precsim x^{* T} \tilde{A} y^{*}$ if and only if

$$
\begin{array}{ll}
x^{T}(A-H) y^{*} \leqslant x^{* T}(A-H) y^{*}, & x^{T} A y^{*} \leqslant x^{* T} A y^{*}, \\
x^{T}(C-L) y^{*} \leqslant x^{* T}(C-L) y^{*}, & x^{T} C y^{*} \leqslant x^{* T} C y^{*}, \\
x^{T}(C+R) y^{*} \leqslant x^{* T}(C+R) y^{*}, & x^{T} C y^{*} \leqslant x^{* T} C y^{*} .
\end{array}
$$

Also, since

$$
x^{* T} \tilde{A} y^{*}=\left(x^{* T} A y^{*}, x^{* T} C y^{*}, x^{* T} B y^{*}, x^{* T} H y^{*}, x^{* T} L y^{*}, x^{* T} R y^{*}, x^{* T} M y^{*}\right),
$$


and

$$
x^{* T} \tilde{A} y=\left(x^{* T} A y, x^{* T} C y, x^{* T} B y, x^{* T} H y, x^{* T} L y, x^{* T} R y, x^{* T} M y\right),
$$

similary by Theorem (2.1), $x^{* T} \tilde{A} y^{*} \precsim x^{* T} \tilde{A} y$ if and only if

$$
\begin{array}{ll}
x^{* T}(A-H) y^{*} \leqslant x^{* T}(A-H) y, & x^{* T} A y^{*} \leqslant x^{* T} A y, \\
x^{* T}(B+M) y^{*} \leqslant x^{* T}(B+M) y, & x^{* T} B y^{*} \leqslant x^{* T} B y, \\
x^{* T}(C-L) y^{*} \leqslant x^{* T}(C-L) y, & x^{* T} C y^{*} \leqslant x^{* T} C y, \\
x^{* T}(C+R) y^{*} \leqslant x^{* T}(C+R) y, & x^{* T} C y^{*} \leqslant x^{* T} C y .
\end{array}
$$

Now, from (3.1) and (3.5) we get

$$
\begin{gathered}
x^{T} A y^{*} \leqslant x^{* T} A y^{*} \leqslant x^{* T} A y, \\
x^{T}(A-H) y^{*} \leqslant x^{* T}(A-H) y^{*} \leqslant x^{* T}(A-H) y,
\end{gathered}
$$

from (3.2) and (3.6) we obtain

$$
\begin{gathered}
x^{T} B y^{*} \leqslant x^{* T} B y^{*} \leqslant x^{* T} B y, \\
x^{T}(B+M) y^{*} \leqslant x^{* T}(B+M) y^{*} \leqslant x^{* T}(B+M) y,
\end{gathered}
$$

from (3.3) and (3.8) we have

$$
\begin{gathered}
x^{T} C y^{*} \leqslant x^{* T} C y^{*} \leqslant x^{* T} C y, \\
x^{T}(C-L) y^{*} \leqslant x^{* T}(C-L) y^{*} \leqslant x^{* T}(C-L) y .
\end{gathered}
$$

and from (3.4) and (3.8) we get

$$
\begin{gathered}
x^{T} C y^{*} \leqslant x^{* T} C y^{*} \leqslant x^{* T} C y, \\
x^{T}(C+R) y^{*} \leqslant x^{* T}(C+R) y^{*} \leqslant x^{* T}(C+R) y .
\end{gathered}
$$

Hence, we have the required inequalities $(i)-(v i i)$.

In the rest of the paper, we purpose the following notations:

$$
A_{0}^{L}=A-H, C_{0}^{L}=C-L, C_{0}^{U}=C+R, B_{0}^{U}=B+M,
$$

where $A, C, B, H, L, R, M$ are the $p \times q$ matrix. Using these notations Theorem(3.1) can be rewrite as follows. 
Corollary 3.1. Let $\tilde{\Gamma}$ be a two-person zero-sum game with fuzzy payoffs, the pair $\left(x^{*}, y^{*}\right)$ is the Nash Equilibrium strategy of $\tilde{\Gamma}$ if and only if the followings hold

$$
\begin{aligned}
& x^{T}\left(A, C, B, A_{0}^{L}, C_{0}^{L}, C_{0}^{U}, B_{0}^{U}\right) y^{*} \leqslant x^{* T}\left(A, C, B, A_{0}^{L}, C_{0}^{L}, C_{0}^{U}, B_{0}^{U}\right) y^{*} \\
& \leqslant x^{* T}\left(A, C, B, A_{0}^{L}, C_{0}^{L}, C_{0}^{U}, B_{0}^{U}\right) y .
\end{aligned}
$$

In the view of Theorem 3.1, we understand that to solve the fuzzy game $\tilde{\Gamma}$, it is enough to consider seven crisp two-person zero-sum games and attempt to determine a point $\left(x^{*}, y^{*}\right) \in X \times Y$ which is simultaneously a saddle point of them.

Definition 3.2. A two-person zero-sum fuzzy game $\tilde{\Gamma}=(X, Y, \tilde{A})$ is called to be a proportional fuzzy game if and only if there exists $\gamma_{n} \in(0,1] ; n=1, \ldots, 4$ such that $h_{i j}=\gamma_{1} a_{i j}, l_{i j}=\gamma_{2} c_{i j}, r_{i j}=\gamma_{3} c_{i j}$ and $m_{i j}=\gamma_{4} b_{i j}$ for all $i=1,2, \ldots, p$ and for all $j=1,2, \ldots, q$.

Theorem 3.2. A pair of mixed strategies $\left(x^{*}, y^{*}\right) \in X \times Y$ is a Nash equilibrium strategy of the proportional fuzzy matrix game $\tilde{\Gamma}=(X, Y, \tilde{A})$ if and only if $\left(x^{*}, y^{*}\right) \in X \times Y$ is the Nash equilibrium of crisp two-person zero-sum games $\Gamma_{a}=(X, Y, A), \Gamma_{b}=(X, Y, B)$ and $\Gamma_{c}=(X, Y, C)$.

Proof. Let $\tilde{\Gamma}=(X, Y, \tilde{A})$ be a proportional fuzzy matrix game. Therefore by Definition (3.2) $\tilde{A}=\left(A, C, B, \gamma_{1} A, \gamma_{2} C, \gamma_{3} C, \gamma_{4} B\right)$ is the payoff matrix of the game. By Theorem $(3.1),\left(x^{*}, y^{*}\right) \in X \times Y$ is a Nash equilibrium of $\tilde{\Gamma}$ if and only if

(i) $x^{T} A y^{*} \leqslant x^{* T} A y^{*} \leqslant x^{* T} A y$,

(ii) $x^{T} B y^{*} \leqslant x^{* T} B y^{*} \leqslant x^{* T} B y$,

(iii) $x^{T} C y^{*} \leqslant x^{* T} C y^{*} \leqslant x^{* T} C y$,

because the other inequalities came to these one. Equivalently, $\left(x^{*}, y^{*}\right) \in X \times Y$ is a Nash equilibrium of crisp two-person zero-sum games $\Gamma_{a}=(X, Y, A), \Gamma_{b}=(X, Y, B)$ and $\Gamma_{c}=(X, Y, C)$. The proof is complete.

The following corollary is a direct result of Theorem(3.2).

Corollary 3.2. Let $\tilde{A}=\left(\tilde{a}_{i j}\right)$ be a payoff matrix of proportional fuzzy game $\tilde{\Gamma}$. Suppose that $b_{i j}=\gamma_{5} a_{i j}, c_{i j}=$ $\gamma_{6} a_{i j}$ for all $i, j$ with $\gamma_{5}, \gamma_{6} \geq 1$. Then a pair of mixed strategies $\left(x^{*}, y^{*}\right) \in X \times Y$ is the Nash equilibrium strategy for $\tilde{\Gamma}$ if and only if $\left(x^{*}, y^{*}\right)$ is a Nash equilibrium of crisp two-person zero-sum game $\Gamma_{a}=(X, Y, A)$.

Definition 3.3. Let $\tilde{\Gamma}$ be a two-person zero-sum fuzzy game. It is called constant fuzzy game if and only if there exist $h, l, r, m>0$ such that $h_{i j}=h, l_{i j}=l, r_{i j}=r$ and $m_{i j}=m$ for all $i=1,2, \ldots, p$ and $j=1,2, \ldots, q$.

Lemma 3.1. Let $\tilde{\Gamma}=(X, Y, \tilde{A})$ be a constant fuzzy game. A pair of mixed strategies $\left(x^{*}, y^{*}\right) \in X \times Y$ is the Nash equilibrium strategy for $\tilde{\Gamma}$ if and only if $\left(x^{*}, y^{*}\right)$ is a Nash equilibrium of crisp two-person zero-sum games $\Gamma_{a}, \Gamma_{b}$ and $\Gamma_{c}$. 
Proof. By Definition(3.3) $H, L, R$ and $M$ are constant matrices which all the entries are $h, l, r$ and $m$, respectively. Hence $x^{T} H y=h, x^{T} L y=l, X^{T} R y=r$ and $x^{T} M y=m$ for all $x \in X, y \in Y$. By Theorem(3.1) the result can be obtained, directly.

Definition 3.4. [3] A pair of mixed strategies $\left(x^{*}, y^{*}\right) \in X \times Y$ is called a Pareto Nash equilibrium strategy of the game $\tilde{\Gamma}$ if

(i) there does not exist any $x \in X$ such that $x^{* T} \tilde{A} y^{*} \precsim x^{T} \tilde{A} y^{*}$,

(ii) there does not exist any $y \in Y$ such that $x^{* T} \tilde{A} y \precsim x^{* T} \tilde{A} y^{*}$.

Theorem 3.3. Let $\tilde{\Gamma} \equiv(X, Y, \tilde{A})$ be a fuzzy two-person zero-sum game. A pair $\left(x^{*}, y^{*}\right) \in X \times Y$ is the Pareto Nash equilibrium strategy for the game $\tilde{\Gamma}$ if and only if

(i) there exist no $x \in X$ such that $x^{* T} A y^{*} \leq x^{T} A y^{*}, x^{* T} B y^{*} \leq x^{T} B y^{*}$, $x^{* T} C y^{*} \leq x^{T} C y^{*}$ and

$$
\begin{aligned}
\left(x^{* T} A_{0}^{L} y^{*}, x^{* T} C_{0}^{L} y^{*}, x^{* T} C_{0}^{U} y^{*}, x^{* T} B_{0}^{U} y^{*}\right) & \leq \\
\left(x^{T} A_{0}^{L} y^{*}, x^{T} C_{0}^{L} y^{*}, x^{T} C_{0}^{U} y^{*}, x^{T} B_{0}^{U} y^{*}\right) & ;
\end{aligned}
$$

(ii) there exist no $y \in Y$ such that $x^{* T} A y \leq x^{T} A y^{*}, x^{* T} B y \leq x^{T} B y^{*}$, $x^{* T} C y \leq x^{T} C y^{*}$ and

$$
\begin{aligned}
&\left(x^{* T} A_{0}^{L} y, x^{* T} C_{0}^{L} y, x^{* T} C_{0}^{U} y, x^{* T} B_{0}^{U} y\right) \leq \\
&\left(x^{* T} A_{0}^{L} y^{*}, x^{* T} C_{0}^{L} y^{*}, x^{* T} C_{0}^{U} y^{*}, x^{* T} B_{0}^{U} y^{*}\right)
\end{aligned}
$$

Proof. By contradiction, let $\left(x^{*}, y^{*}\right) \in X \times Y$ be the Pareto Nash equilibrium strategy of $\tilde{\Gamma}$. Assume that there exist $x_{1} \in X$ such that following relationships are established

$$
\left(x^{* T} A_{0}^{L} y^{*}, x^{* T} C_{0}^{L} y^{*}, x^{* T} C_{0}^{U} y^{*}, x^{* T} B_{0}^{U} y^{*}\right) \leq\left(x_{1}^{T} A_{0}^{L} y^{*}, x_{1}^{T} C_{0}^{L} y^{*}, x_{1}^{T} C_{0}^{U} y^{*}, x_{1}^{T} B_{0}^{U} y^{*}\right),
$$

and

$$
x^{* T} A y^{*} \leq x_{1}^{T} A y^{*}, x^{* T} B y^{*} \leq x_{1}^{T} B y^{*}, x^{* T} C y^{*} \leq x_{1}^{T} C y^{*}
$$

It implies that

$$
\begin{aligned}
& x^{* T} A_{0}^{L} y^{*} \leq x_{1}^{T} A_{0}^{L} y^{*}, x^{* T} C_{0}^{L} y^{*} \leq x_{1}^{T} C_{0}^{L} y^{*}, \\
& x^{* T} C_{0}^{U} y^{*} \leq x_{1}^{T} C_{0}^{U} y^{*}, x^{* T} B_{0}^{U} y^{*} \leq x_{1}^{T} B_{0}^{U} y^{*} .
\end{aligned}
$$

But, by Definition (2.3) the above inequalities do not occur simultaneously. Therefore, we get 


$$
\begin{aligned}
\left(x^{* T}\left(\frac{\alpha}{k} A+\left(1-\frac{\alpha}{k}\right)(A-H)\right) y^{*}, x^{* T}\left(\frac{\alpha}{k} B+\left(1-\frac{\alpha}{k}\right)(B+M)\right) y^{*}\right) \leq \\
\left(x_{1}^{T}\left(\frac{\alpha}{k} A+\left(1-\frac{\alpha}{k}\right)(A-H)\right) y^{*}, x_{1}^{T}\left(\frac{\alpha}{k} B+\left(1-\frac{\alpha}{k}\right)(B+M)\right) y^{*}\right),
\end{aligned}
$$

for $\alpha \in[0, k]$ and obtain

$$
\begin{aligned}
\left.\left(x^{* T}\left(\frac{\alpha-k}{1-k}\right) C+\left(1-\frac{\alpha-k}{1-k}\right)(C-L)\right) y^{*}, x^{* T}\left(\frac{\alpha-k}{1-k} C+\left(1-\frac{\alpha-k}{1-k}\right)(C+R)\right) y^{*}\right) \leq \\
\left.\left(x_{1}^{T}\left(\frac{\alpha-k}{1-k}\right) C+\left(1-\frac{\alpha-k}{1-k}\right)(C-L)\right) y^{*}, x_{1}^{T}\left(\frac{\alpha-k}{1-k} C+\left(1-\frac{\alpha-k}{1-k}\right)(C+R)\right) y^{*}\right),
\end{aligned}
$$

for $\alpha \in[k, 1]$. By rearranging, it follows that

$$
\begin{aligned}
\left(x^{* T}\left(A-\left(1-\frac{\alpha}{k}\right) H\right) y^{*}, x^{* T}\left(B+\left(1-\frac{\alpha}{k}\right) M\right) y^{*}\right) & \leq \\
& \left(x_{1}^{T}\left(A-\left(1-\frac{\alpha}{k}\right) H\right) y^{*}, x_{1}^{T}\left(B+\left(1-\frac{\alpha}{k}\right) M\right) y^{*}\right),
\end{aligned}
$$

and

$$
\begin{aligned}
&\left(x^{* T}\left(C-\left(\frac{1-\alpha}{1-k}\right) L\right) y^{*}, x^{* T}\left(C+\left(\frac{1-\alpha}{1-k}\right) R\right) y^{*}\right) \leq \\
&\left(x_{1}^{T}\left(C-\left(\frac{1-\alpha}{1-k}\right) L\right) y^{*}, x_{1}^{T}\left(C+\left(\frac{1-\alpha}{1-k}\right) R\right) y^{*}\right) .
\end{aligned}
$$

Using Definition (3.4) it implies that $x^{* T} \tilde{A} y^{*} \preceq x_{1}^{T} \tilde{A} y^{*}$. This is a contradiction.

Conversely, we assume that the pair of mixed strategy $\left(x^{*}, y^{*}\right) \in X \times Y$ be satisfy (3.9) and (3.10). Suppose that there exists a strategy $x_{1} \in X$ such that $x^{* T} \tilde{A} y^{*} \preceq x_{1}^{T} \tilde{A} y^{*}$. By Definition 2.4, we have for all $\alpha \in[0,1]$

$$
\left(x^{* T} A_{\alpha}^{L} y^{*}, x^{* T} A_{\alpha}^{U} y^{*}\right) \leq\left(x_{1}^{T} A_{\alpha}^{L} y^{*}, x_{1}^{T} A_{\alpha}^{U} y^{*}\right)
$$

which

$$
\left(A-\left(1-\frac{\alpha}{k}\right) H\right)=A_{\alpha}^{L},\left(B+\left(1-\frac{\alpha}{k}\right) M\right)=A_{\alpha}^{U} \text { for } \alpha \in[0, k]
$$

and

$$
\left(C-\left(\frac{1-\alpha}{1-k}\right) L\right)=A_{\alpha}^{L},\left(C+\left(\frac{1-\alpha}{1-k}\right) R\right)=A_{\alpha}^{U} \text { for } \alpha \in[k, 1] .
$$

Set $\alpha=0$, then

$$
x^{* T}\left(A_{0}^{L}, C_{0}^{L}, C_{0}^{U}, B_{0}^{U}\right) y^{*} \leq x_{1}^{T}\left(A_{0}^{L}, C_{0}^{L}, C_{0}^{U}, B_{0}^{U}\right) y^{*}
$$

and

$$
x^{* T} A y^{*} \leq x^{T} A y^{*}, x^{* T} B y^{*} \leq x^{T} B y^{*}, x^{* T} C y^{*} \leq x^{T} C y^{*} .
$$

This is contradict (i). Similarly, we can show that there does not exist any $y \in Y$ such that $x^{* T} \tilde{A} y \preceq x^{* T} \tilde{A} y^{*}$. Then proof of the Theorem is complete. 
Definition 3.5. A pair of mixed strategies $\left(x^{*}, y^{*}\right) \in X \times Y$ is a weak Pareto Nash equilibrium strategy of the game $\tilde{\Gamma}$ if

(i) there does not exist any $x \in X$ such that $x^{* T} \tilde{A} y^{*} \prec x^{T} \tilde{A} y^{*}$,

(ii) there does not exist any $y \in Y$ such that $x^{* T} \tilde{A} y \prec x^{* T} \tilde{A} y^{*}$.

Following theorem is obtaine directly from Definition (3.5) and Theorem (3.3).

Theorem 3.4. Let $\tilde{\Gamma} \equiv(X, Y, \tilde{A})$ be a fuzzy two-person zero-sum game. A pair $\left(x^{*}, y^{*}\right) \in X \times Y$ is the weak Pareto Nash equilibrium strategy for the game $\tilde{\Gamma}$ if and only if

(i) there exist no $x \in X$ such that

$$
\begin{aligned}
&\left(x^{* T} A_{0}^{L} y^{*}, x^{* T} C_{0}^{L} y^{*}, x^{* T} C_{0}^{U} y^{*}, x^{* T} B_{0}^{U} y^{*}\right)< \\
&\left(x^{T} A_{0}^{L} y^{*}, x^{T} C_{0}^{L} y^{*}, x^{T} C_{0}^{U} y^{*}, x^{T} B_{0}^{U} y^{*}\right)
\end{aligned}
$$

and

$$
x^{* T} A y^{*}<x^{T} A y^{*}, x^{* T} B y^{*}<x^{T} B y^{*}, x^{* T} C y^{*}<x^{T} C y^{*}
$$

(ii) there exist no $y \in Y$ such that

$$
\begin{aligned}
&\left(x^{* T} A_{0}^{L} y, x^{* T} C_{0}^{L} y, x^{* T} C_{0}^{U} y, x^{* T} B_{0}^{U} y\right)< \\
&\left(x^{* T} A_{0}^{L} y^{*}, x^{* T} C_{0}^{L} y^{*}, x^{* T} C_{0}^{U} y^{*}, x^{* T} B_{0}^{U} y^{*}\right)
\end{aligned}
$$

and

$$
x^{* T} A y<x^{T} A y^{*}, x^{* T} B y<x^{T} B y^{*}, x^{* T} C y<x^{T} C y^{*} .
$$

\section{Parametric Bi-Matrix Games}

In this section we characterize parametric bi-matrix games and investigate other types of Nash equilibrium strategies for parametric bi-matrix games. Let $S^{p}=\left\{\eta_{1}, \eta_{2}, \ldots, \eta_{p}\right\}$ and $S^{q}=\left\{\xi_{1}, \xi_{2}, \ldots, \xi_{q}\right\}$ be sets of pure strategies of player $I$ and player $I I$, respectively. We set $U=\left(u_{i j}\right)_{p \times q}$ to be payoffs matrices of player $I$ and $V=\left(V_{i j}\right)_{p \times q}$ to be payoffs matrices of player $I I$, respectively. Suppose $\beta, \gamma \in[0,1]$ and let $(1-\beta)\left(a_{i j}+c_{i j}-h_{i j}-l_{i j}\right)+\beta\left(c_{i j}+r_{i j}+b_{i j}+m_{i j}\right)$ be the gain of player $I$ and $(1-\gamma)\left(a_{i j}+c_{i j}-h_{i j}-\right.$ $\left.l_{i j}\right)+\gamma\left(c_{i j}+r_{i j}+b_{i j}+m_{i j}\right)$ be the losses of player $I I$ when player $I$ emploing pure strategy $i$ and player II emploing pure strategy $j$. Then the game $\Gamma=(X, Y, U, V)$ is called a bi-matrix game. The notation of parametric as follow;

Suppose $\beta, \gamma \in[0,1]$, then we set

$$
A(\beta)=(1-\beta)(A+C-H-L)+\beta(C+R+B+M),
$$


and

$$
-A(\gamma)=-[(1-\gamma)(A+C-H-L)+\gamma(C+R+B+M)] .
$$

Now, we consider the parametric bi-matrix game $\Gamma(\beta, \gamma)=(X, Y, A(\beta), A(\gamma))$.

Definition 4.1. [8] Let $\Gamma(\beta, \gamma)$ be a parametric bi-matrix game. A pair of mixed strategies $\left(x^{*}, y^{*}\right) \in X \times Y$ is a Nash equilibrium strategy of $\Gamma$ if

(i) $x^{T} A(\beta) y^{*} \leq x^{* T} A(\beta) y^{*}$ for all $x \in X$,

(ii) $x^{* T} A(\gamma) y^{*} \leq x^{* T} A(\gamma) y$ for all $y \in Y$.

Theorem 4.1. Let $\Gamma(\beta, \gamma)$ be a prametric bi-matrix game and the pair of mixed strategy $\left(x^{*}, y^{*}\right) \in X \times Y$ be Nash equilibrium strategy of $\Gamma$. Then $\left(x^{*}, y^{*}\right) \in X \times Y$ is the Pareto Nash equilibrium strategy of the fuzzy two-person zero-sum game $\tilde{\Gamma}$.

Proof. Let $\left(x^{*}, y^{*}\right) \in X \times Y$ be the Nash equilibrium strategy of the parametric bi-matrix game $\Gamma(\beta, \gamma)$, which $\beta, \gamma \in[0,1]$. By Definition (4.1) we obtain

$$
\begin{aligned}
& (1-\beta) x^{T}(A+C-H-L) y^{*}+\beta x^{T}(C+R+B+M) y^{*} \leq \\
& (1-\beta) x^{* T}(A+C-H-L) y^{*}+\beta x^{* T}(C+R+B+M) y^{*},
\end{aligned}
$$

and

$$
\begin{aligned}
(1-\gamma) x^{* T}(A+C-H-L) y^{*}+\gamma x^{* T}(C+R+B+M) y^{*} \leq \\
(1-\gamma) x^{* T}(A+C-H-L) y+\gamma x^{* T}(C+R+B+M) y
\end{aligned}
$$

In order to show that $\left(x^{*}, y^{*}\right) \in X \times Y$ is Pareto Nash equilibrium strategy of $\tilde{\Gamma}$, we have to prove that there exist $x_{1} \in X$ such that $x^{* T} \tilde{A} y^{*} \preceq x_{1}^{T} \tilde{A} y^{*}$ holds. From Definition (2.4), we get

$$
\begin{aligned}
&\left(x^{* T} A_{0}^{L} y^{*}, x^{* T} C_{0}^{L} y^{*}, x^{* T} C_{0}^{U} y^{*}, x^{* T} B_{0}^{U} y^{*}\right) \leq \\
&\left(x_{1}^{T} A_{0}^{L} y^{*}, x_{1}^{T} C_{0}^{L} y^{*}, x_{1}^{T} C_{0}^{U} y^{*}, x_{1}^{T} B_{0}^{U} y^{*}\right) .
\end{aligned}
$$

Moreover, by Definition (2.3)

$$
\begin{aligned}
& x^{* T} A_{0}^{L} y^{*}=x_{1}^{T} A_{0}^{L} y^{*}, \quad x^{* T} C_{0}^{L} y^{*}=x_{1}^{T} C_{0}^{L} y^{*}, \\
& x^{* T} C_{0}^{U} y^{*}=x_{1}^{T} C_{0}^{U} y^{*}, \quad x^{* T} B_{0}^{U} y^{*}=x_{1}^{T} B_{0}^{U} y^{*},
\end{aligned}
$$

do not occur simultaneously. Then we have

$$
\begin{aligned}
(1-\beta) x^{* T}(A+C-H-L) y^{*}+\beta x^{* T}(C+R+B+M) y^{*}< \\
(1-\beta) x_{1}^{T}(A+C-H-L) y^{*}+\beta x_{1}^{T}(C+R+B+M) y^{*} .
\end{aligned}
$$


This is a contradiction. The condition (ii) can de proved, similarly.

Theorem 4.2. Let the pair of mixed strategies $\left(x^{*}, y^{*}\right) \in X \times Y$ be Nash equilibrium strategy of prametric bi-matrix game $\Gamma(\beta, \gamma)$ with $\beta, \gamma \in[0,1]$. Then $\left(x^{*}, y^{*}\right) \in X \times Y$ is the weak Pareto Nash equilibrium strategy of fuzzy two-person zero-sum game $\tilde{\Gamma}$.

The following corollary is direct result of Theorem (4.1) and Theorem (4.2).

Corollary 4.1. A fuzzy two-person zero-sum game $\tilde{\Gamma}$ satisfies the following properties:

(i) There exsist at least one Pareto Nash equilibrium strategy of fuzzy game $\tilde{\Gamma}$,

(ii) There exsist at least one weak Pareto Nash equilibrium strategy of fuzzy game $\tilde{\Gamma}$.

\section{Illustrative Examples}

Example 5.1. Let $\tilde{\Gamma}$ be a fuzzy two-person zero-sum game and $\tilde{A}$ be the fuzzy payoff matrix of $\tilde{\Gamma}$ given as follows:

$$
\tilde{A}=\left(\begin{array}{cc}
(20,40,60,2,8,12,24) & (70,140,210,7,28,42,84) \\
(50,100,150,5,20,30,60) & (10,20,30,1,4,6,12)
\end{array}\right) .
$$

Find the Nash equilibrium strategy for the game $\tilde{\Gamma}$.

Obviously, $\tilde{\Gamma}$ is a proportional fuzzy game. Note that $\gamma_{1}=0.1, \gamma_{2}=0.2, \gamma_{3}=0.3$ and $\gamma_{4}=0.4$. Let $x^{* T}=(p, 1-p)$ and $y^{* T}=(q, 1-q)$ be the mixed strategy of player I and II, respectively. By theorem $(3.2)$, the Nash equilibrium strategy of game $\tilde{\Gamma}$ can be obtined by solving a bi-matrix game whose payoff matrices are

$$
A=\left(\begin{array}{cc}
20 & 70 \\
50 & 10
\end{array}\right), C=\left(\begin{array}{cc}
40 & 140 \\
100 & 20
\end{array}\right), B=\left(\begin{array}{cc}
60 & 210 \\
150 & 30
\end{array}\right)
$$

We have

$$
\left(\begin{array}{ll}
1 & 0
\end{array}\right)\left(\begin{array}{cc}
20 & 70 \\
50 & 10
\end{array}\right)\left(\begin{array}{c}
q \\
1-q
\end{array}\right) \leq\left(\begin{array}{ll}
p & 1-p
\end{array}\right)\left(\begin{array}{cc}
20 & 70 \\
50 & 10
\end{array}\right)\left(\begin{array}{c}
q \\
1-q
\end{array}\right)
$$

and

$$
\left(\begin{array}{ll}
p & 1-p
\end{array}\right)\left(\begin{array}{cc}
20 & 70 \\
50 & 10
\end{array}\right)\left(\begin{array}{c}
q \\
1-q
\end{array}\right) \leq\left(\begin{array}{ll}
p & 1-p
\end{array}\right)\left(\begin{array}{ll}
20 & 70 \\
50 & 10
\end{array}\right)\left(\begin{array}{l}
0 \\
1
\end{array}\right)
$$

It is easy to obtain that the Nash equilibrium strategy of the crisp matrix game $\Gamma_{a}$ is $\left(x^{*}, y^{*}\right)=$ $\left(\left(\frac{4}{9}, \frac{5}{9}\right),\left(\frac{2}{3}, \frac{1}{3}\right)\right)$ and similarly the Nash equilibrium strategy of the crisp matrix games $\Gamma_{b}$ and $\Gamma_{c}$ can be obtained. So expected value of the gasme $\tilde{\Gamma}$ is

$$
\left(\frac{4}{9}, \frac{5}{9}\right) \tilde{A}\left(\frac{2}{3}, \frac{1}{3}\right)^{T}=\left(\frac{990}{27}, \frac{1980}{27}, \frac{2870}{27}, \frac{99}{27}, \frac{396}{27}, \frac{594}{27}, \frac{1188}{27}\right) .
$$


Example 5.2. Let $\tilde{A}$ be the payoff matrix of the fuzzy two-person zero-sum game $\tilde{\Gamma}$, given as follows:

$$
\tilde{A}=\left(\begin{array}{cc}
(50,100,150,10,15,10,40) & (80,160,240,10,15,10,40) \\
(100,200,300,10,15,10,40) & (20,40,60,10,15,10,40)
\end{array}\right) .
$$

Find the Nash equilibrium strategy for the game $\tilde{\Gamma}$.

by definition(3.3) $\quad \tilde{\Gamma}$ is a proportional fuzzy game and $h=10, l=15, r=10$ and $m=40$. Let $x^{* T}=(p, 1-p)$ and $y^{* T}=(q, 1-q)$ be the mixed strategy of player I and II, respectively. By Theorm $(3.1)$, it is easy to show that the Nash equilibrium strategy of $\tilde{\Gamma}$ is $\left(x^{*}, y^{*}\right)=\left(\left(\frac{8}{11}, \frac{3}{11}\right),\left(\frac{6}{11}, \frac{5}{11}\right)\right)$ and the expected value of $\tilde{\Gamma}$ is given by

$$
\left(\frac{8}{11}, \frac{3}{11}\right) \tilde{A}\left(\frac{6}{11}, \frac{5}{11}\right)^{T}=\left(\frac{7700}{121}, \frac{15400}{121}, \frac{2310}{121}, 10,15,10,40\right)
$$

Example 5.3. Consider the fuzzy two-person zero-sum game $\tilde{\Gamma}$ with heptagonal fuzzy payoff matrix $\tilde{A}$ given by

$$
\tilde{A}=\left(\begin{array}{cc}
(90,100,120,10,5,10,15) & (70,80,100,15,5,10,20) \\
(60,90,100,15,10,5,10) & (170,180,210,20,5,20,10)
\end{array}\right)
$$

Find the Nash, Pareto Nash and weak Pareto Nash equilibrium strategy of the game $\tilde{\Gamma}$.

Let $x^{* T}=(p, 1-p)$ and $y^{* T}=(q, 1-q)$ be the mixed strategy of player I and II, respectively. Since there is no $(x, y) \in X \times Y$ satisfying the conditions of Theorem(3.1), so there is no Nash equilibrium strategy for the game $\tilde{\Gamma}$. By Theorem(4.2) to finding the Pareto Nash equilibrium strategy, it is enough to find the Nash equilibrium strategy of parametric bi-matrix game $\tilde{\Gamma}$. So, we construct the bi-matrix game $\Gamma(\beta, \gamma)$ from fuzzy matrix game $\tilde{\Gamma}$. Using relations (4.1) and (4.2) we obtain

$$
A(\beta)=\left(\begin{array}{ll}
175+70 \beta & 130+80 \beta \\
125+80 \beta & 325+95 \beta
\end{array}\right), A(\gamma)=\left(\begin{array}{ll}
175+70 \gamma & 130+80 \gamma \\
125+80 \gamma & 325+95 \gamma
\end{array}\right)
$$

where $\beta, \gamma \in[0,1]$. It is easy to see that $\left(x^{*}, y^{*}\right)$ is the Nash equilibrium strategy for the parametric bi-matrix game $\Gamma(\beta, \gamma)$ if it satisfies the following:

$$
\begin{array}{ll}
(1,0) A(\beta) y^{*} \leq x^{* T} A(\beta) y^{*}, & (0,1) A(\beta) y^{*} \leq x^{* T} A(\beta) y^{*}, \\
x^{* T} A(\gamma) y^{*} \leq x^{* T} A(\gamma)(0,1)^{T}, & x^{* T} A(\gamma) y^{*} \leq x^{* T} A(\gamma)(1,0)^{T},
\end{array}
$$

which are equivalent to

$$
\left\{\begin{array}{l}
(245+5 \beta)(1-p) q-(195+15 \beta)(1-p) \leq 0 \\
(245+5 \beta) p q-(195+15 \beta) p \geq 0
\end{array}\right.
$$




$$
\left\{\begin{array}{l}
(245+5 \gamma)(1-q) p-(200+15 \gamma)(1-q) \geq 0 \\
(245+5 \gamma) p q-(200+15 \gamma) q \leq 0
\end{array}\right.
$$

Thus for $\beta, \gamma \in[0,1]$, Nash equilibrium strategy for the parametric game $\Gamma(\beta, \gamma)$ are as follows

$$
\left(x_{1}^{*}, x_{2}^{*}\right)=\left(\frac{200+15 \gamma}{245+5 \gamma}, \frac{45-10 \gamma}{245+5 \gamma}\right),\left(y_{1}^{*}, y_{2}^{*}\right)=\left(\frac{195+15 \beta}{245+5 \beta}, \frac{50-10 \beta}{245+5 \beta}\right) .
$$

Therfore by Theorem(4.1) and (4.2) the Pareto Nash and weak Pareto Nash equilibrium strategy of the game $\tilde{\Gamma}$ are as following

$$
\begin{aligned}
& \left\{\left(x^{*}, y^{*}\right)^{T}=\left(\left(\frac{200+15 \gamma}{245+5 \gamma}, \frac{45-10 \gamma}{245+5 \gamma}\right),\left(\frac{195+15 \beta}{245+5 \beta}, \frac{50-10 \beta}{245+5 \beta}\right)\right) \mid \beta, \gamma \in[0,1]\right\}, \\
& \left\{\left(x^{*}, y^{*}\right)^{T}=\left(\left(\frac{200+15 \gamma}{245+5 \gamma}, \frac{45-10 \gamma}{245+5 \gamma}\right),\left(\frac{195+15 \beta}{245+5 \beta}, \frac{50-10 \beta}{245+5 \beta}\right)\right) \mid \beta, \gamma \in(0,1)\right\},
\end{aligned}
$$

respectively.

\section{REFERENCES}

[1] C. R. Bector, S. Chandra, Fuzzy Mathematical Programming and Fuzzy Matrix Games, springer, Berlin, 2005.

[2] L. Campos, Fuzzy linear programming model to solve fuzzy matrix game, Fuzzy Sets Syst., 32(3)(1989), $275-289$.

[3] Bapi Dutta, S. K. Gupta, On Nash equilibrium strategy of two-person zero-sum games with Trapezoidal fuzzy payoffs, Fuzzy Inf. Eng., 6(3)(2014), 299-314.

[4] Li Cunlin, Zhang Qiang, Nash equilibrium strategy for fuzzy non-cooperative games, Fuzzy Sets Syst., 176(1)(2011), 46-55.

[5] B. Liu, Uncertain programming. New York:wiley, 1999.

[6] B. Liu , Uncertainty theory. An introduction to its axiomatic foundations, Studies in Fuzziness and Soft Computing, 154, Springer-Verlag, Berlin, 2004.

[7] T. Maeda ,On characterization of equilibrium strategy of bimatrix games with fuzzy payoffs, J. Math. Anal. Appl., $251(2)(2000), 885-896$.

[8] J. F Nash, Non-cooperative games, Ann. Math, 54(2)(1951), 286-295.

[9] J. von Neumann, O. Morgenstern, Theory of Games and Economic Behavior, Princeton University Press, Princeton, New Jersey, 1944.

[10] K. Rathi, S. Balamohan, Representation and Ranking of fuzzy numbers with Heptagonal membership function using value and Ambiguity index, Appl. Math. Sci., 87(8)(2014), 4309-4321.

[11] J. Ramik, J. R imanek, Inequality relation between fuzzy numbers and its use in fuzzy optimization, Fuzzy Sets Syst., 16(2)(1985), 123-138.

[12] M. Sakawa, Fuzzy Sets and Interactive Multiobjective Optimization, Plenum Press, New York, 1993.

[13] A. V. Yazenin, Fuzzy and stochastic programming, Fuzzy Sets Syst., 22(1-2)(1987), 171-180.

[14] L. A. Zadeh, Fuzzy sets, Information and Control, (8)(1965), 338-353.

[15] L. A. Zadeh, Fuzzy set as a basis for a theory of possibility, Fuzzy Sets Syst., 1(1978), 3-28.

[16] H. J. Zimmermann, Application of fuzzy set theory to mathematical programming, Inform. Sci., 36(1-2)(1985), 29-58.

[17] H. J. Zimmermann, Fuzzy Set Theory and its Applications, Second edition, Kluwer Academic Publishers, Boston, MA, 1992. 